\title{
Cost Analysis: Use of Hydrogel Plug Tract Sealant System for CT-Guided Lung Biopsy can Reduce Patient Recovery Time and Cost
}

\author{
Lee Shimwoo* \\ Department of Radiology, Ronald Reagan UCLA Medical Center, USA
}

*Corresponding author: Lee Shimwoo, Department of Radiology, Ronald Reagan UCLA Medical Center, Los Angeles, CA, USA.

To Cite This Article: Lee Shimwoo, Cost Analysis: Use of Hydrogel Plug Tract Sealant System for CT-Guided Lung Biopsy can Reduce Patient Recovery Time and Cost. Am J Biomed Sci \& Res. 2021 - 11(5). AJBSR.MS.ID.001670. DOI: 10.34297/AJBSR.2021.11.001670.

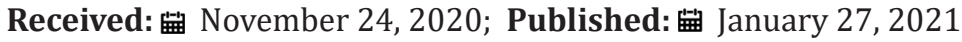

\begin{abstract}
Purpose: To evaluate whether the use of the BioSentry hydrogel plug after a lung biopsy can reduce costs by safely shortening post-procedure observation time.

Materials and methods: A single-center, retrospective review of percutaneous CT-guided core needle lung biopsies utilizing the hydrogel plug from May 2013-July 2016 was performed. Post-procedurally, patients were observed either for 3 hours with chest radiographs obtained at 1 and 3 hours, or for 1.5 hours with one chest radiograph. Biopsy-related cost data was supplied from the institution's finance department.

Results: A total of 235 patients were analyzed for pneumothorax rates (124 patient in 3-hour recovery group and 111 patients the 1.5-hour group). Pneumothorax rate on follow-up chest radiographs was $22 \%$ vs $13 \%$ in the 3-hour vs 1.5 -hour recovery groups, and chest tube insertion rate was 3.9\% vs $0 \%$, respectively. In a 30-day follow-up available in $96 \%$ of patients, the 3 - and 1.5 -hour groups each had 1 patient hospitalized for delayed complications but neither required chest tube placement. Discharging patients if absent or small stable pneumothorax detected on 1.5-hour post-lung biopsy recovery chest radiograph resulted in total cost savings of $\$ 686(\mathrm{P}<0.05)$ and recovery cost savings of $\$ 487(\mathrm{P}<0.001)$ on average per patient.
\end{abstract}

Conclusion: Hydrogel plug use and early discharge at 1.5 hours resulted in significant cost savings. 1.5-hour observation is safe for routine lung biopsies, while a longer observation is recommended for complicated patients or technically challenging biopsies.

Keywords: Lung biopsy; Cost; Hydrogel plug

\section{Introduction}

Demand for lung biopsy is increasing with rising lung cancer rates, higher detection rates of incidental pulmonary nodules, and greater need for tissue sampling for new molecular testing [1]. CT-guided percutaneous core needle lung biopsy is the standard method for evaluating pulmonary lesions with 93-95\% diagnostic accuracy [2-4]. Pneumothorax is the most common complication of the procedure, with incidence of approximately 20-25\%, with 4-8\% requiring chest tube placement [5-7]. Complications of lung biopsy can be a substantial economic burden, increasing costs by $300-400 \%[8,9]$. There is great interest in reducing pneumothorax rates and lowering potential costs related to chest tube placement, additional imaging, hospital admission and stay.

Sealing the pleural puncture site after lung biopsy has shown to decrease pneumothorax rates. Various sealant materials have been investigated, including hydrogel plug, autologous blood path, gelatin sponge slurry or plug, fibrin glue and saline. One of the best studied sealants is the polyethylene glycol hydrogel plug (BioSentryTM tract sealant system, AngioDynamics, Latham, NY), which works by expanding on contact with moisture and sealing the biopsy tract. This sealant system was the first device to be approved 
by the Food and Drug Administration to reduce the incidence of pneumothoraces following CT-guided lung biopsy. As such, multiple studies have demonstrated that the hydrogel plug decreased both pneumothorax rates $(18-20 \%$ vs $31-33 \%)[10,11]$ and chest tube insertion rates ( $2 \%$ vs $10 \%$ ) [12] compared to no sealant.

While clinical practices vary by institution and interventionalist, patients are typically monitored after routine lung biopsy for pneumothoraces with serial chest radiographs during recovery. The post-procedural recovery period, often charged based on time, can be a significant portion of the lung biopsy costs. Given the efficacy of the hydrogel plug in reducing the risk of post-biopsy pneumothoraces, the aim of this study was to retrospectively evaluate if length of post biopsy recovery can be safely shortened with use of such a device at our institution.

\section{Methods}

\section{Study subjects and selection criteria}

After approval from the institutional review board (IRB \#16000217), we retrospectively reviewed electronic medical records and images of all percutaneous CT-guided core needle lung biopsies performed at our institution utilizing the BioSentry device from May 2013-July 2016. A total of 285 biopsies were performed utilizing the BioSentry hydrogel plug. Of these patients, 50 were excluded from our observational analysis for the following reasons: inpatient status (32), requiring chest tube placement [13], known BioSentry maldeployment during procedure [3], patient age $<18$ years at time of biopsy [1], and pre-existing hydropneumothorax [1]. Therefore, a total of uncomplicated 235 core needle lung biopsies were available for further review.

\section{Procedures and assessments}

Three board-certified, fellowship-trained interventional radiologists (with 24,12, and 8 years of experience) performed biopsies in a single institution. Using CT-guidance, 20-gauge core samples were percutaneously obtained via 19-gauge coaxial needle (Argon Medical Devices, Athens, Tex). The BioSentry hydrogel plug tract sealant was deployed through the same coaxial system using the technique previously described by Zaetta et al. [10].

Post-procedurally, 124 patients were observed for 3 hours during which two portable upright chest radiographs were obtained at 1 and 3 hours. An additional 111 patients were observed for a shorter duration of 1.5 hours with a single portable chest radiograph (Figure 1). A decrease in the length of recovery time reflected the evolving practice of the interventionalists over time at our institution, with a larger portion of biopsies with short recovery stay occurring during the later dates of the cohort sampling period. Chart review was performed to assess for biopsyrelated delayed complications with a total follow-up time of 30 days (mean 30 days).

\section{Study outcomes and statistical analysis}

Presence of pneumothorax on immediate post-procedure CT imaging was defined as none, non-actionable (small), or requiring chest tube. Post-procedure radiographs were also assessed for pneumothorax, which was defined as none, small if pleural air accumulation was $<2 \mathrm{~cm}$ from lung apex, and large if pleural air accumulation was $>2 \mathrm{~cm}$ from lung apex [13].

Biopsy-related cost data was supplied from our institution's finance department. Our EPSi costing system database was queried for all activity codes associated with the lung biopsy encounter. Itemized cost and charge obtained for the biopsy encounter was obtained. The charge was defined as the amount asked by provider and was what appeared on medical bill, whereas the cost was the amount the insurance company and/or patient actually paid for health care services [14]. Total cost was defined as the sum of costs of all activity codes associated with the encounter. Recovery cost was defined as fee for post-procedure observation nursing care, charged per 15-minute intervals at our institution. Of the 235 total uncomplicated biopsy patients, 145 (62\%) had available data for cost analysis, (53\% and $79 \%$ of the 3 -hour and 1.5 -hour recovery groups, respectively).

Mann-Whitney $\mathrm{U}$ and independent $\mathrm{t}$ tests were performed for continuous nonparametric and parametric variables. Pearson chi squared tests were performed for categorical data. $\mathrm{P}<0.05$ was considered significant.

\section{Results}

\section{Patient demographics}

A total of 235 patients who underwent core needle lung biopsies were reviewed (Table 1). The mean patient age at time of biopsy was 67 years. Overall, there were even proportions of male and female ( $52 \%$ male) patients. The average nodule mean diameter was $19 \mathrm{~mm}$, ranging from $3.5-94 \mathrm{~mm}$. The average nodule depth from pleural surface, measured from nodule periphery along needle shaft to pleural surface just prior to biopsy, was $39 \mathrm{~mm}$. Emphysema was present in the same lobe as the nodule in $16 \%$ of the biopsies. There was no statistically significant difference in these variables between the 3 - and 1.5-hour recovery groups.

\section{Pneumothorax rates}

Analysis of pneumothorax rates included 11 of the 13 patients who required chest tube placement for pneumothorax. The other 2 chest tube patients were not included because pneumothoraces occurred after pleural puncture but before BioSentry deployment. The pneumothorax rate after BioSentry use on post-procedural chest radiographs was 18\% (44/246) and the chest tube placement rate was $4.5 \%$ (11/246), comparable to prior studies on BioSentry, ranging from $15-30 \%$ for pneumothorax and $2-8 \%$ for chest tube 
insertion (10-12). For comparison between the 3- and 1.5-hour recovery groups, additional 7 chest tube patients were removed from analysis given that they developed significant pneumothoraces on immediate post-procedural CT scans after BioSentry deployment, prompting chest tube placement immediately. Pneumothorax rate on follow-up radiographs was $22 \%$ vs $13 \%$ in the 3 - and 1.5 -hour recovery groups, and chest tube insertion rate was $3.9 \%$ vs $0 \%$, respectively (Table 2).

Table 1: Lung biopsy patient demographics, comparison of 3- versus 1.5-hour post-procedure recovery groups.

\begin{tabular}{|c|c|c|c|c|}
\hline Characteristic & Total Patients & BioSentry 3hour Recovery & BioSentry 1.5hour Recovery & Statistical Significance \\
\hline Number of patients & 235 & 124 & 111 & - \\
\hline $\begin{array}{c}\text { Age, mean } \pm \text { SD1 (range), } \\
\text { years }\end{array}$ & $66.9 \pm 11.7(27-94)$ & $66.9 \pm 12.0(32-94)$ & $67.1 \pm 11.3(27-92)$ & $P=0.87$ \\
\hline Sex, male, n (\%) & $121(51.5 \%)$ & $59(47.6 \%)$ & $62(55.9 \%)$ & $P=0.21$ \\
\hline $\begin{array}{l}\text { Nodule size: mean diam- } \\
\text { eter, mean } \pm \text { SD (range), } \\
\mathrm{mm}\end{array}$ & $18.8 \pm 13.1(3.5-94)$ & $18.1 \pm 11.2(3.5-68.5)$ & $19.5 \pm 15.0(4.5-94)$ & $\mathrm{P}=0.97$ \\
\hline $\begin{array}{l}\text { Nodule size: longest } \\
\text { diameter, mean } \pm \text { SD } \\
\text { (range), mm }\end{array}$ & $21.1 \pm 14.4(4-109)$ & $20.8 \pm 13.1(4-78)$ & $21.5 \pm 15.8(5-109)$ & $P=0.89$ \\
\hline $\begin{array}{l}\text { Depth from pleural sur- } \\
\text { face, mean } \pm \text { SD (range) } \\
\mathrm{mm}\end{array}$ & $38.5 \pm 17.8(6-92)$ & $41.0 \pm 18.9(13-92)$ & $35.6 \pm 16.1(6-82)$ & $P=0.08$ \\
\hline $\begin{array}{l}\text { Emphysema in lobe of } \\
\text { biopsy, } n(\%)\end{array}$ & $37(15.7 \%)$ & $22(17.7 \%)$ & $15(13.5 \%)$ & $P=0.38$ \\
\hline
\end{tabular}

1SD: Standard Deviation

Table 2: Post-procedure pneumothorax rate comparison of 3-hour versus 1.5-hour post-procedure recovery groups.

\begin{tabular}{|c|c|c|c|}
\hline Post procedure Pneumothorax & Total Patients & BioSentry 3hour Recovery & BioSentry 1.5hour Recovery \\
\hline Pneumothorax on CT à Pneumothorax on CXR1 & 239 & 128 & 111 \\
\hline None $\diamond$ None & $158(67.2 \%)$ & $85(66.4 \%)$ & $73(65.8 \%)$ \\
\hline Small $\diamond$ Small & $29(12.3 \%)$ & $15(11.7 \%)$ & $14(12.6 \%)$ \\
\hline Small $\diamond$ None & $31(13.2 \%)$ & $15(11.7 \%)$ & $16(14.4 \%)$ \\
\hline None $\diamond$ Small & $10(4.2 \%)$ & $8(6.3 \%)$ & $2(1.8 \%)$ \\
\hline None | Small $\diamond$ Large & $5(3.9 \%)$ & $5(3.9 \%)^{*}$ & $0(0 \%)$ \\
\hline
\end{tabular}

${ }^{1}$ CXR: Chest Radiograph, * 4 out of 5 patients underwent chest tube placement and were excluded for subsequent cost analysis

Table 3: Cost analysis of lung biopsy procedure, comparison of 3-hour versus 1.5-hour post-procedure recovery groups.

\begin{tabular}{|c|c|c|c|c|c|}
\hline Characteristic & Total patients & BioSentry 3 hour recovery & BioSentry 1.5 hour recovery & Average Savings & Statistical Significance \\
\hline Total Charge & $\$ 8,670.42$ & $\$ 9,448.46$ & $\$ 8,020.42$ & $\$ 1,428.04$ & $\mathrm{P}<0.001$ \\
\hline (mean \pm SD1) & $\pm 2,389.88$ & $\pm 2,293.67$ & $\pm 2,284.75$ & $\$ 686.47$ & $\mathrm{P}=0.034$ \\
\hline Total Cost & $\$ 4,781.60$ & $\$ 5,155.61$ & $\$ 4,469.14$ & \\
\hline (mean \pm SD) & $\pm 1,942.39$ & $\pm 2,051.03$ & $\pm 1,800.68$ & $\$ 871.72$ & $\mathrm{P}<0.001$ \\
\hline Recovery Charge & $\$ 1,157.79$ & $\$ 1,632.73$ & \pm 761.01 & & \\
\hline (mean \pm SD) & \pm 564.72 & \pm 416.16 & $\$ 608.67$ & & $\mathrm{P}<0.001$ \\
\hline Recovery Cost & $\$ 829.61$ & $\$ 1,095.69$ & \pm 210.69 & \\
\hline (mean \pm SD) & \pm 347.81 & \pm 292.46 & & & \\
\hline
\end{tabular}

1SD: Standard Deviation

8 out of 128 (6.3\%) patients observed for 3 hours had no pneumothoraces on immediate post-procedure CT scans but developed small pneumothoraces on follow-up chest radiograph. 5 patients $(3.9 \%)$ had no or small pneumothoraces on CT but developed large pneumothoraces, of which 4 underwent chest tube placement. One patient did not require chest tube placement since the pneumothorax was stable on follow-up imaging. Of those requiring chest tubes, 2 patients did not demonstrate enlarging pneumothoraces until the second post-procedure chest radiograph. For 1 of the 2 patients, the biopsy needle was repositioned multiple times, eventually requiring a second coaxial needle placement. For the second patient, a mediastinal biopsy was performed spanning 
the right middle lobe, with the BioSentry plug unable to adequately cross both pleural punctures (Figure 2). 2 out of 111 (1.8\%) patients observed for 1.5 hours had no pneumothoraces on CT but developed small pneumothoraces on follow-up radiograph. These pneumothoraces were thought to be so trace in size that these patients were sent home at 1.5 hours. No patients developed large pneumothorax on follow-up radiographs. Multivariate analysis of patient and biopsy characteristics for developing a pneumothorax, including patient age, nodule size and depth from pleura and emphysema, showed no statistically significant associations in both the 3- and 1.5-hour groups.

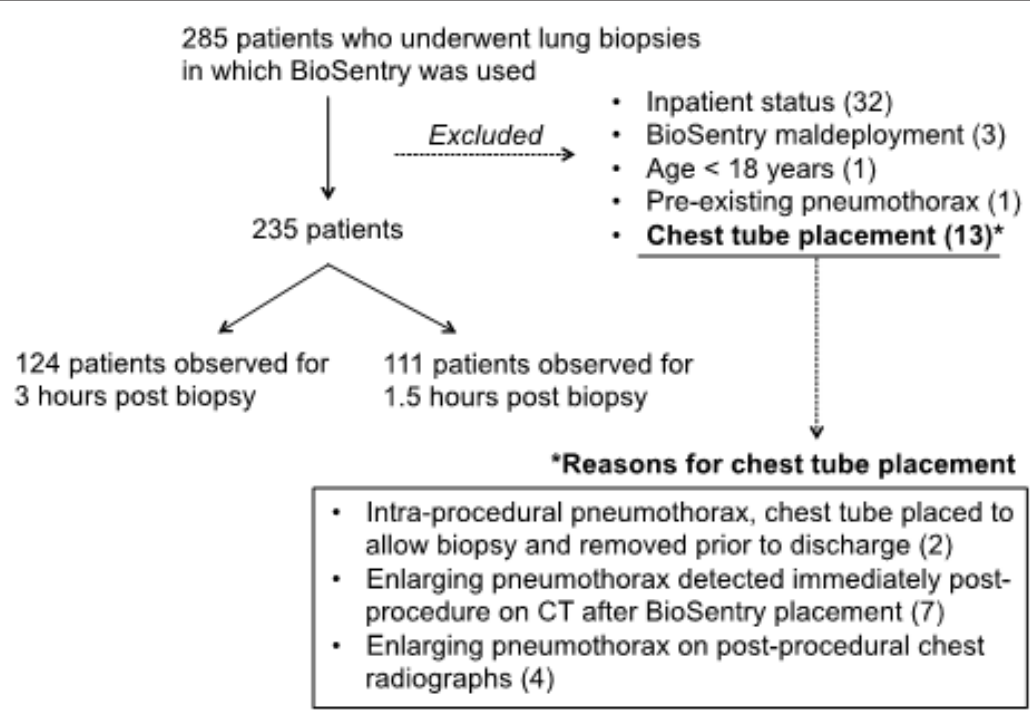

Figure 1: Costs were compared between the 3- and 1.5-hour recovery groups, excluding chest tube placement patients.

Complications within 30 days of biopsy were compared between the 3-hour and 1.5-hour recovery groups (Figure 3) In the 3-hour recovery group, potential delayed biopsy-related complications were noted in 6 patients, including transient mild hemoptysis (3), worsened dyspnea (2), and chest pain (1). Of these 6 patients, 4 had emphysema and 1 had history of known lung adenocarcinoma. One patient who had severe emphysema and had a small pneumothorax on immediate post-biopsy CT scan that remained stable on follow-up radiographs presented to the emergency room for dyspnea 1 day after biopsy. The patient was hospitalized and treated conservatively without a chest tube. In the 1.5-hour recovery group, delayed complications were noted in 4 patients, including transient mild hemoptysis (2), worsened dyspnea (1), and pneumothorax (1). The delayed pneumothorax was noted in a patient with metastatic neuroectodermal tumor who developed worsening shortness of breath 1 week after a mediastinal biopsy, requiring hospital admission but without chest tube placement. The other patients with delayed complications also had history of known malignancy at the time of biopsy, 2 with primary lung cancer and 1 with metastatic renal cell carcinoma.

\section{Cost Analysis}

The costs and charges were compared between the 3- and 1.5-hour recovery groups, excluding chest tube placement patients (Figure 1). In this study, we mainly focused on costs for our analyses since it is likely a more accurate reflection of what is generally referred to as "health care costs" incurred to the society. The average total cost in the 1.5 -hour recovery group was $\$ 4,469$ ( \pm $1,801)$ compared to $\$ 5,156( \pm 2,051)$ in the 3-hour recovery group, resulting in average cost savings of $\$ 686(\mathrm{P}<0.05)$ (Table 3). The recovery cost was decreased by $\$ 487(\mathrm{P}<0.001)$ in the 1.5 -hour group. The use of BioSentry did not add significantly to the overall cost of the procedure due to the use of c-code for billing approved by the Center for Medicare and Medicaid Services to offset the cost of the device, as well as institutionally negotiated price.

\section{Discussion}

In our single-center retrospective study, we examined whether the use of a hydrogel sealant system after a lung biopsy can safely reduce costs by shortening post-procedure observation time. Discharging routine lung biopsy patients in the absence of or a small stable pneumothorax after a 1.5-hour recovery resulted in total cost savings of $\$ 686(\mathrm{P}<0.05)$ on average. Specifically, recovery cost was reduced by $\$ 487(\mathrm{P}<0.001)$. At our institution, observation nursing care is charged by 15 -minute intervals, with the cost estimate of $\$ 85$ every 15 minutes. Based on this, the expected cost savings of reducing observation time from 3 to 1.5 hours is $\$ 510$, which is comparable to the actual cost saved.

There is strong interest in reducing costs related to lung biopsy. Biopsy is the most expensive step in lung cancer diagnosis, and complications are not only detrimental to patients' health but can 
also further drive up medical costs up to four-fold [9]. Patients who require chest tube insertion after biopsy are more likely to develop respiratory failure requiring mechanical ventilation and have longer lengths of hospital stay [15]. A recent study by MarcoDomenech et al. observed that using hydrogel plug for inpatient lung biopsies is cost-effective as it reduces pneumothorax rates and length of hospital stay [16]. To our knowledge, our study is the first in the literature to demonstrate that post-biopsy observation time can be safely reduced with the use of hydrogel plugs and result in significant cost savings.

Thoughtful reduction of post-biopsy observation stay has additional benefits. With shorter observation, lung biopsies can be safely added later in the day and at the end of the workweek, which are time slots that many operators do not routinely schedule lung biopsies in case of pneumothorax and chest tube insertion. Recently, with much interest focused on moving elective procedures from inpatient to outpatient settings, the ability to perform higher number of lung biopsies per day would be cost-attractive and impose less scheduling restrictions. Earlier studies have shown that discharge as early as 30 minutes after lung biopsy without use of sealant system is feasible with a chest drain insertion rate of $7-11 \%[17,18]$. For comparison, chest tube insertion rate in our cohort was $4 \%$ after BioSentry deployment. Sealant systems like BioSentry are able to provide interventionalists greater confidence in the clinical course of their patients by lowering adverse events, therefore making the argument for shortening recovery times even more compelling.

Ensuring patient safety while reducing observation time is crucial. Isolated risk pools were detected in our patient cohort where longer observation time is warranted. These include enlarging pneumothorax on initial recovery chest film indicative of an active air leak, as well as complicated or technically challenging procedures, such as mediastinal biopsies and multiple pleural punctures. Repeated pleural puncture is a well-established risk factor for development of post-biopsy pneumothorax [19]. Long needle path or increased depth of the lesion from the skin $(>4 \mathrm{~cm})$ has also been associated with an increased risk [18,19] although such association was not appreciated on this study. Emphysema is another important risk factor known to increase pneumothorax rates. Of note, an abstract by Hoffman et al. found that patients with emphysema have higher rates of pneumothorax when using BioSentry compared to no sealant [20] although the mechanism is unclear. Presence of emphysema was less common in our biopsy cohort, only occurring in $15.7 \%$ of patients. The pneumothorax rate of these patients was approximately $20 \%$, not significantly different from the overall rate of $18 \%$.

Our study had several limitations. More patients underwent shorter recovery in the later dates of data collection, which encaptures a period of evolving clinical practice at our institution. Although there were no demonstrable differences in patient demographics between the 3- and 1.5-hour recovery groups, we could not account for all aspects of clinical decisions that determined recovery stay length given the retrospective nature of our study. The finding of $0 \%$ pneumothorax rate in the 1.5-hour group may be due to such bias and/or due to not large enough sample size. However, the results of the 30-day follow-up help show that the longer recovery times do not necessarily lead to more detection of clinically significant pneumothoraces. Another limitation is the lack of a control group without the use of BioSentry. In addition, cost data was missing in 38\% of patients. The reasons for missing data are not fully elucidated although they may be related to patients' involvement in other research trials. Future prospective randomized studies on a larger scale are needed to further establish safe post-lung biopsy observation protocols.

\section{References}

1 Marshall D, Laberge JM, Firetag B, Miller T, Kerlan RK (2013) The changing face of percutaneous image-guided biopsy: Molecular profiling and genomic analysis in current practice. J Vasc Interv Radiol 24(8): 1094-1103.

2 WangY,JiangF, TanX, Tian P (2016) CT-guided percutaneous transthoracic needle biopsy for paramediastinal and nonparamediastinal lung lesions: Diagnostic yield and complications in 1484 patients. Medicine 95(31): e4460.

3 Takeshita J, Masago K, Kato R, Hata A, Kaji R, et al. (2015) CT-guided fine-needle aspiration and core needle biopsies of pulmonary lesions: A single-center experience with 750 biopsies in Japan. Am J Roentgenol 204(1): 29-34.

4 Yang W, Sun W, Li Q, Yao Y, Lv T, et al. (2015) Diagnostic accuracy of CTguided transthoracic needle biopsy for solitary pulmonary nodules. PLoS One 10(6): e0131373.

5 Covey AM, Gandhi R, Brody LA, Getrajdman G, Thaler HT, et al. (2004) Factors Associated with Pneumothorax and Pneumothorax Requiring Treatment after Percutaneous Lung Biopsy in 443 Consecutive Patients. J Vasc Interv Radiol 15(5): 479-483.

6 Moreland A, Novogrodsky E, Brody L, Durack J, Erinjeri J, et al. (2016) Pneumothorax with prolonged chest tube requirement after CT-guided percutaneous lung biopsy: incidence and risk factors. Eur Radiol 26: 3483-3491.

7 Maybody M, Muallem N, Brown KT, Moskowitz CS, Hsu M, et al. Autologous Blood Patch Injection versus Hydrogel Plug in CT-guided Lung Biopsy: A Prospective Randomized Trial. Radiology 290(2): 547-554.

8 Gurley MB, Richli WR, Waugh KA (1998) Outpatient management of pneumothorax after fine-needle aspiration: economic advantages for the hospital and patient. Radiology 209(3): 717-722.

9 Lokhandwala T, Bittoni MA, Dann RA, D’Souza AO, Johnson M, et al. (2017) Costs of Diagnostic Assessment for Lung Cancer: A Medicare Claims Analysis. Clin Lung Cancer 18(1): e27-e34.

10 Zaetta JM, Licht MO, Fisher JS, Avelar RL (2010) A lung biopsy tract plug for reduction of postbiopsy pneumothorax and other complications: Results of a prospective, multicenter, randomized, controlled clinical study. J Vasc Interv Radiol 21(8): 1235-1243.

11 Ahrar JU, Gupta S, Ensor JE, Mahvash A, Sabir SH, et al. (2017) Efficacy of a Self-expanding Tract Sealant Device in the Reduction of Pneumothorax 
and Chest Tube Placement Rates After Percutaneous Lung Biopsy: A Matched Controlled Study Using Propensity Score Analysis. Cardiovasc Intervent Radiol 40(2): 270-276.

12 Grage RA, Keogh S, Naveed MA: Comparison analysis pre and post implementation of a BioSentry tract sealant system after percutaneous transthoracic CT guided needle biopsy. J Vasc Interv Radiol 2015; 26:S149.

13 MacDuff A, Arnold A, Harvey J (2010) Management of spontaneous pneumothorax: British Thoracic Society pleural disease guideline 2010. Thorax 65: ii18-ii31.

14 Arora V, Moriates C, Shah N (2015) The challenge of understanding health care costs and charges. AMA J Ethics 17(11): 1046-1052.

15 Wiener RS, Schwartz LM, Woloshin S, Gilbert Welch H (2011) Populationbased risk for complications after transthoracic needle lung biopsy of a pulmonary nodule: An analysis of discharge records. Ann Intern Med 155(3): 137-144.

16 Marco-Doménech SF, Fernández-García P, Navarro-Ballester A, CifriánPérez M, Escobar-Valero, et al. (2019) Cost-effectiveness of hydrogel plugs in CT-guided lung biopsies. Radiologia 61(2): 153-160.
17 Dennie CJ, Matzinger FR, Marriner JR, Maziak DE (2001) Transthoracic needle biopsy of the lung: Results of early discharge in 506 outpatients. Radiology 219(1): 247-251.

18 Tavare AN, Creer DD, Khan S, Vancheeswaran R, Hare SS (2016) Ambulatory percutaneous lung biopsy with early discharge and Heimlich valve management of iatrogenic pneumothorax: More for less. Thorax 71(2): 190-192.

19 Moore EH, Shepard JA, McLoud TC, Templeton PA, Kosiuk JP (1990) Positional precautions in needle aspiration lung biopsy. Radiology 175(3): 733-735.

20 Hoffman C, White E, Wilson J, Dickey K, Durran R, et al. (2019) 03:54 PM Abstract No. 409 Impact of a pleural tract sealant system on pneumothorax rates after lung biopsy at an academic institution. J Vasc Interv Radiol 6: S99-S107. 